\title{
Lower geriatric nutritional risk index predicts postoperative pancreatic fistula in patients with distal pancreatectomy
}

\author{
NAOTAKE FUNAMIZU, YUKIO NAKABAYASHI and KAZUNAO KURIHARA \\ Department of Digestive Surgery, Kawaguchi Municipal Medical Center, Kawaguchi, Saitama 333-0833, Japan
}

Received March 21, 2019; Accepted October 22, 2019

DOI: $10.3892 /$ mco.2019.1960

\begin{abstract}
Postoperative pancreatic fistula (POPF) is a common complication following pancreatic resection. It leads to increased medical costs, and longer hospital stays. However, the risk factors of POPF are still unclear, and therefore, this urgent clinical issue should be resolved. The geriatric nutritional risk index (GNRI) is tool to assess the nutritional status using body weight, and serum albumin value, particularly in elderly patients. On the other hand, POPF is associated with body mass index (BMI). Thus, the present study aimed to investigate whether GNRI can predict the risk of POPF in patients after distal pancreatectomy (DP). We conducted a retrospective cohort study involving 37 patients who were treated at the Department of Digestive Surgery, Kawaguchi Municipal Medical Center between January 2007 and June 2018. All patients were subjected to nutritional screening using GNRI, and were followed up after DP for postoperative complications including POPF. In addition, risk factors of POPF, and patient's height, BMI, and preoperative laboratory values were analyzed. POPF was observed in 7 of the $37(19 \%)$ patients. Those with a POPF had significantly lower GNRI values than those without POPF $(\mathrm{P}<0.001)$. Receiver operating characteristic curve analysis was performed to determine a cut-off value of GNRI, which indicated an increased risk of POPF. This value was determined as 96 (sensitivity: 71.4\%, specificity: 86.7\%, likelihood ratio: 5.36). Univariate analysis confirmed that a GNRI of $<96$ was significantly associated with POPF $(\mathrm{P}=0.005)$, and a multivariate logistic regression analysis revealed that a GNRI of $<96$ was significant independent predictor of POPF $(\mathrm{P}=0.005)$, suggesting its utility for assessing the risk of POPF following DP.
\end{abstract}

Correspondence to: Dr Naotake Funamizu, Department of Digestive Surgery, Kawaguchi Municipal Medical Center, 180 Nishi-araijuku, Kawaguchi, Saitama 333-0833, Japan

E-mail: funamizujikei@yahoo.co.jp

Key words: distal pancreatectomy, geriatric nutritional risk index, postoperative pancreatic fistula

\section{Introduction}

Distal pancreatectomy (DP) is the standard procedure for tumors located in the body or tail of the pancreas, such as pancreatic carcinoma, intraductal papillary mucinous neoplasm, neuroendocrine neoplasm, and invasive carcinomas from other organ carcinomas including stomach and colon (1). A common complication of DP is postoperative pancreatic fistula (POPF). Despite advances in surgical techniques and devices, incidence of POPF over the last few decades have been $24-40 \%(2,3)$. Moreover, perioperative morbidity rates associated with POPF of $30 \%$ have been reported (4). POPF has the potential to cause lethal intra-abdominal abscess and bleeding (4). POPF increases medical costs and prolong hospital stays. Peng et al reported that body mass index (BMI), blood transfusion, intraoperative blood loss, and prolonged operative time are clinical predictors of POPF in patients who undergo DP (5). The geriatric nutritional risk index (GNRI) is a tool that has been proposed to evaluate nutrition-related risks in elderly patients (6) and can be easily calculated from body weight, height, and serum albumin levels. Due to the intimate relationship between preoperative body weight and POPF (5), we examined whether GNRI is a potential tool to predict POPF in patients who undergo DP.

\section{Patients and methods}

Patients. Between January 2007 and June 2018, 37 patients underwent DP for pancreatic tumors or invasive gastric cancer at the Kawaguchi Municipal Medical Center (Kawaguchi, China). We retrospectively analyzed the records of these patients. The exclusion criteria were as follows: i) invasion to celiac artery; ii) peritoneal dissemination and iii) difficulty of radical resection by DP. The present study protocol was reviewed and approved by the Ethics Committee of the Kawaguchi Municipal Medical Center in 2016. All patients or their guardians consented to the use of their medical information for scientific research (ethics approval no. KMMC2017-27). The requirement for informed consent from all participants was waived because of the retrospective nature of the present study. They had all undergone DP with splenectomy, and the pancreatic remnant had been closed with a stapler.

Clinicopathological data. Data collected from the medical records included the occurrence of POPF, demographic 
variables (sex and age), anthropometric parameters (height, weight, and BMI), comorbidities, American Society of Anesthesiologist (ASA)'s physical status classification, blood transfusions, estimated blood loss, operative time, and serum albumin levels. POPF was classified according to the International Study Group of Pancreatic Fistula definition and grading (A, B, or C) (7). Grade A indicates asymptomatic POPF, whereas, grades $\mathrm{B}$ and $\mathrm{C}$ are symptomatic and require intervention, such as antibiotics therapies and/or drainage for grade $\mathrm{B}$, and resuscitation and/or exploratory laparotomy for grade $\mathrm{C}$ fistulas. Drain amylase was monitored on post-operative day $1,3,5$, and 7 .

Nutritional assessment using GNRI. Preoperative nutritional status was assessed with GNRI, which was calculated as GNRI $=[14.89 \mathrm{x}$ serum albumin $(\mathrm{g} / \mathrm{l})]+[41.7 \mathrm{x}$ actual/ideal body weight $(\mathrm{kg})]$. Ideal body weight was calculated as ideal body weight $=$ the patient's height $(\mathrm{m}) \times$ height $(\mathrm{m}) \times 22(\mathrm{BMI})$. When the actual body weight was higher than the ideal weight, the ratio was set to 1 .

These heights are all actual height. This formula was used for male and female in the same way.

Statistical analysis. All statistical analyses were performed using Graphpad Prism v5.0 (Graphpad Software Inc.) and StatView (Abacus Concepts, Inc.). Differences between the patients with and without POPF were compared using the Fisher's exact test or Chi-square test. The optimal cut-off value of GNRI was determined using a receiver operating characteristic (ROC) curve. The potential risk factors of POPF were evaluated using univariate and multivariate analyses. Univariate analysis was conducted using the Chi-square or Fisher's exact test, followed by multivariate analysis using a logistic regression to identify the risk factors of POPF. Results are shown as odds ratios and $95 \%$ confidence intervals. $\mathrm{P}<0.05$ was considered to indicate a statistically significant difference.

\section{Results}

Patient characteristics. Of the 37 patients that were reviewed, 22 were men and 15 were women. The median age was 73 (range 35-82) years. POPF occurred in seven (19\%) patients (grade B in all). There were no statistically significant differences between patients with POPF and those without POPF with respect to sex, age, BMI, diabetes mellitus history, operative time, estimated blood loss, blood transfusions, or the presence of soft pancreas. In addition, malignant tumor did not affect risk of POPF (Table I). However, preoperative serum albumin levels and GNRI were significantly lower in patients with POPF than those without POPF $(\mathrm{P}<0.001$, for both variables).

Calculation of optimal GNRI cut-off value. The area under the curve (ROC) was 0.90 (Fig. 1). A GNRI of 96 was determined using Youden index as the appropriate cut-off value that had a sensitivity of $71.4 \%$, a specificity of $86.7 \%$ and a likelihood ratio of 5.36. Patients were categorized into two groups: Group A (GNRI $\geq 96, n=28)$ and group B (GNRI <96, n=9). POPF was observed in $7.1 \%$ of patients in group A and $55.6 \%$ of patients in group B.

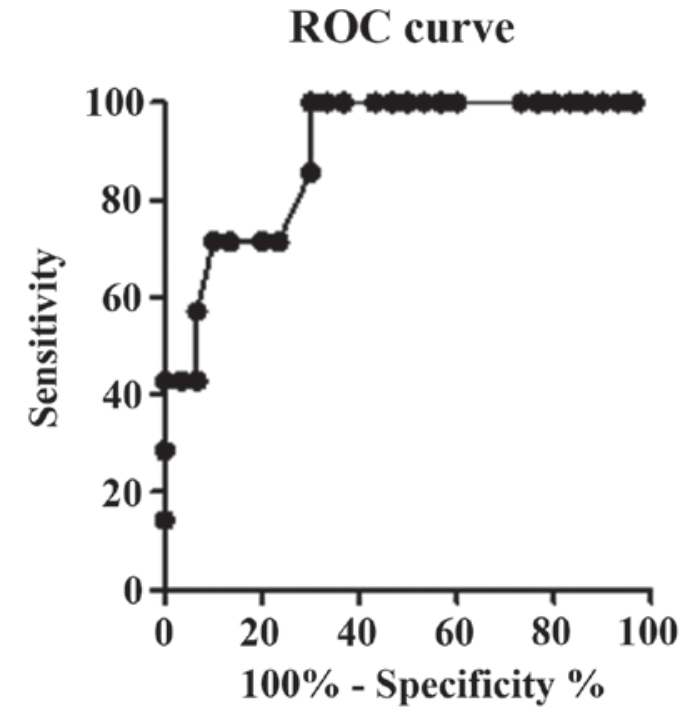

Figure 1. Selection of the GNRI cut-off value. A GNRI of 96 was selected as an optimal cut-off value with sensitivity $71.4 \%$ and specificity $86.7 \%$. GNRI, geriatric nutritional risk index; ROC, receiver operating characteristic.

Univariate and multivariable analyses. Univariate analysis was performed to evaluate factors predicting the risk of POPF after DP. Compared with patients with GNRI $\geq 96$, the incidence of POPF was significantly higher in patients with GNRI <96 ( $\mathrm{P}=0.005)$. Similarly, logistic regression analysis revealed that a GNRI $<96$ was an independent predictor of POPF (P=0.005; Table II).

\section{Discussion}

POPFs remain a major pancreas-specific postoperative complication after pancreatic resection, occurring in approximately $30-40 \%$ of patients after DP (3). A number of risk factors of POPF following pancreatic resection have been recognized, including a soft pancreas, obesity, blood transfusion, age, sex, diabetes mellitus history, preoperative serum albumin levels, extended lymphadenectomy, substantial intraoperative blood loss, and longer operative time (8). At the tissue level, substantial inflammatory activity, indicated by high levels of proinflammatory cytokines and matrix metalloproteinases, and marked chronic inflammatory infiltrates are reportedly associated with a lower incidence of fistula (9). A meta-analysis indicated that a soft pancreas, high BMI, blood transfusion, intraoperative blood loss, and operative time were clinical predictors of POPF (5). Of these variables, a higher than normal $\mathrm{BMI}$ is the most common risk factor of POPF after DP and pancreaticoduodenectomy (PD) $(5,10,11)$. Therefore, preoperative body weight including nutritional status should be improved to avoid postoperative complications. As POPFs are associated with a prolonged hospital stay, ultimately leading to higher medical costs, surgeons should promptly identify at-risk patients prior to surgery. Considering this, several methods of nutritional assessment including body weight have been developed and validated: The malnutrition inflammation score, nutritional risk index (NRI), prognostic nutritional index (PNI), and GNRI (6,12-14). Among them, GNRI was originally developed to evaluate malnutrition and 
Table I. Patients were divided by presence of absence of POPF. The data exhibited a higher incidence of POPF in GNRI $<96$ compared with GNRI $\geq 96$ patients.

\begin{tabular}{lccc}
\hline Characteristics & POPF group, $\mathrm{n}=7$ & Non-POPF group, $\mathrm{n}=30$ & P-value \\
\hline Male/female & $3 / 4$ & $17 / 13$ & 0.68 \\
Age, years & $72.0 \pm 1.8$ & $70.3 \pm 1.9$ & 0.67 \\
BMI & $22.7 \pm 1.8$ & $22.4 \pm 1.9$ & 0.88 \\
Diabetes mellitus: Yes (\%) & $4(57.1)$ & $13(43.3)$ & 0.68 \\
Preoperative albumin, g/l & $3.2 \pm 0.3$ & $4.2 \pm 0.1$ & $<0.001$ \\
GNRI & $85.8 \pm 6.8$ & $103.5 \pm 1.2$ & 4 \\
<96 & 5 & 26 & 0.001 \\
$\geq 96$ & 2 & $31 / 8$ & 0.005 \\
Malignant/benign tumor & $6 / 1$ & $1414.0 \pm 282.1$ & 0.15 \\
Time of operation, min & $342.4 \pm 17.7$ & $12(40.0)$ & 0.40 \\
Estimated blood loss, ml & $814.3 \pm 216.6$ & $27(90.0)$ & 0.32 \\
Blood transfusion: Yes $(\%)$ & $1(14.3)$ & $2(6.7)$ & 0.22 \\
Soft pancreas: Yes $(\%)$ & $5(71.4)$ & $7(100.0)$ & 0.23 \\
SSIs: Yes (\%) & & $<0.001$ \\
\hline
\end{tabular}

POPF, postoperative pancreatic fistula; GNRI, geriatric nutritional risk index; BMI, body mass index; SSIs, surgical site infections.

Table II. Multivariate analysis by logistical regression demonstrated that GNRI <96 was an independent risk factor to predict POPF following DP.

\begin{tabular}{lccc}
\hline Characteristics & Odds ratio & $95 \%$ CI & P-value \\
\hline BMI & 0.99 & $0.83-1.18$ & 0.87 \\
Preoperative albumin, g/l & 19.9 & $2.41-165.25$ & 0.006 \\
GNRI <96 & 0.062 & $0.009-0.43$ & 0.005 \\
\hline
\end{tabular}

GNRI, geriatric nutritional risk index; BMI, body mass index; POPF, postoperative pancreatic fistula; DP, distal pancreatectomy; CI, confidence interval.

related morbidity and mortality in elderly patients (6). Our previous study showed that a low GNRI was associated with wound infections and POPF in patients who undergo PD (15). Therefore, we hypothesized that GNRI could be predictive of POPF following DP. In the present study, $19 \%$ of the 37 patients developed POPF following DP at our hospital. Malignant tumors did not affect the risk of POPF as with Sierzega et al who reported that malnutirition was associated with POPF risk (16). For these patients, GNRI <96 was strongly associated with a higher risk of POPF, suggesting that correcting nutrition before the operation might decrease the risk. In addition, POPF is also a potential predictor of surgical site infection and is intimately related particularly to organ/space infections. Parikh et al reported that $55 \%$ of POPFs contributed to the occurrence of intra-abdominal abscess (17). In our data, all patients with POPF had surgical site infections, with $85.7 \%$ having organ/space infections. However, in present study, blood transfusion was not associated with POPF. We estimated the reason why were that our sample was small, and criteria of judgement for transfusion were different according to anesthesiologists in our hospital.
Our findings are consistent with those of previous studies, in which NRI was associated with POPF following DP (16). Moreover, Sato et al concluded that low PNI was significantly more common in patients with grade B or C POPF (18). Our study has several limitations. The results should be regarded with caution because of the relatively sample size. A further limitation is the retrospective nature of this study. Therefore, a larger prospective study should be conducted to validate the present findings. In conclusions, the present study revealed that a GNRI score of $<96$ may be a risk factor of POPF in patients who undergo DP.

\section{Acknowledgements}

The authors would like to thank Dr Noriko Funamizu (Department of Internal Medicine, Hirose Hospital) for their advice and discussion.

\section{Funding}

No funding was received 


\section{Availability of data and materials}

The datasets used and analyzed during the present study are available from the corresponding author on reasonable request.

\section{Authors' contributions}

NF carried out the experimental studies, and drafted and completed the manuscript. NF and KK participated in the design of the study. YN performed the surgical procedure with NF and revised the manuscript. NF conceived the study and performed the statistical analysis. All authors read and approved the final manuscript.

\section{Ethics approval and consent to participate}

The present study was conducted based on the Declaration of Helsinki after receiving approval from the review board of the Kawaguchi Municipal Medical Center (ethics approval no. KMMC2017-27). The requirement for informed consent from the participants was waived because of the retrospective nature of the present study.

\section{Patient consent for publication}

Not applicable.

\section{Competing interests}

The authors declare that they have no competing interests.

\section{References}

1. Parikh PY and Lillemoe KD: Surgical management of pancreatic cancer-distal pancreatectomy. Semin Oncol 42: 110-122, 2015.

2. van Hilst J, de Pastena M, de Rooij T, Alseidi A, Busch OR, van Dieren S, van Eijck CH, Giovinazzo F, Groot Koerkamp B, Marchegiani G, et al: Clinical impact of the updated international postoperative pancreatic fistula definition in distal pancreatectomy. HPB (Oxford) 20: 1044-1050, 2018.

3. Diener MK, Knaebel HP, Witte ST, Rossion I, Kieser M, Buchler MW, Seiler CM and DISPACT Trial Group: DISPACT trial: A randomized controlled trial to compare two different surgical techniques of DIStal PAnCreaTectomy-study rationale and design. Clin Trials 5: 534-545, 2008.

4. Diener MK, Seiler CM, Rossion I, Kleeff J, Glanemann M, Butturini G, Tomazic A, Bruns CJ, Busch OR, Farkas S, et al: Efficacy of stapler versus hand-sewn closure after distal pancreatectomy (DISPACT): A randomised, controlled multicentre trial. Lancet 377: 1514-1522, 2011.

5. Peng YP, Zhu XL, Yin LD, Zhu Y, Wei JS, Wu JL and Miao Y: Risk factors of postoperative pancreatic fistula in patients after distal pancreatectomy: A systematic review and meta-analysis. Sci Rep 7: 185, 2017.
6. Bouillanne O, Morineau G, Dupont C, Coulombel I, Vincent JP, Nicolis I, Benazeth S, Cynober L and Aussel C: Geriatric nutritional risk index: A new index for evaluating at-risk elderly medical patients. Am J Clin Nutr 82: 777-783, 2005.

7. Bassi C, Dervenis C, Butturini G, Fingerhut A, Yeo C, Izbicki J, Neoptolemos J, Sarr M, Traverso W, Buchler M and International Study Group on Pancreatic Fistula Definition: Postoperative pancreatic fistula: An international study group (ISGPF) definition. Surgery 138: 8-13, 2005.

8. Goh BK, Tan YM, Chung YF, Cheow PC, Ong HS, Chan WH, Chow PK, Soo KC, Wong WK and Ooi LL: Critical appraisal of 232 consecutive distal pancreatectomies with emphasis on risk factors, outcome, and management of the postoperative pancreatic fistula: A 21-year experience at a single institution. Arch Surg 143: 956-965, 2008.

9. Felix K, Schuck A, Gaida MM, Hinz U, Dovzhanskiy D and Werner J: Objective parameters aid the prediction of fistulas in pancreatic surgery. Exp Ther Med 8: 719-726, 2014.

10. Kimura W, Miyata H, Gotoh M, Hirai I, Kenjo A, Kitagawa Y, Shimada M, Baba H, Tomita N, Nakagoe T, et al: A pancreaticoduodenectomy risk model derived from 8575 cases from a national single-race population (Japanese) using a web-based data entry system: The 30-day and in-hospital mortality rates for pancreaticoduodenectomy. Ann Surg 259: 773-780, 2014.

11. Sell NM, Pucci MJ, Gabale S, Leiby BE, Rosato EL, Winter JM, Yeo CJ and Lavu H: The influence of transection site on the development of pancreatic fistula in patients undergoing distal pancreatectomy: A review of 294 consecutive cases. Surgery 157: 1080-1087, 2015.

12. Kalantar-Zadeh K, Kopple JD, Block G and Humphreys MH: A malnutrition-inflammation score is correlated with morbidity and mortality in maintenance hemodialysis patients. Am J Kidney Dis 38: 1251-1263, 2001.

13. Adejumo OL, Koelling TM and Hummel SL: Nutritional risk index predicts mortality in hospitalized advanced heart failure patients. J Heart Lung Transplant 34: 1385-1389, 2015.

14. Hu Q, Wang G, Ren J, Ren H, Li G, Wu X, Gu G, Li R, Guo K, Deng Y, et al: Preoperative prognostic nutritional index predicts postoperative surgical site infections in gastrointestinal fistula patients undergoing bowel resections. Medicine (Baltimore) 95: e4084, 2016.

15. Funamizu N, Nakabayashi Y, Iida T and Kurihara K: Geriatric nutritional risk index predicts surgical site infection after pancreaticoduodenectomy. Mol Clin Oncol 9: 274-278, 2018.

16. Sierzega M, Niekowal B, Kulig J and Popiela T: Nutritional status affects the rate of pancreatic fistula after distal pancreatectomy: A multivariate analysis of 132 patients. J Am Coll Surg 205: 52-59, 2007.

17. Parikh JA, Beane JD, Kilbane EM, Milgrom DP and Pitt HA: Is American college of surgeons NSQIP organ space infection a surrogate for pancreatic fistula? J Am Coll Surg 219: 1111-1116, 2014.

18. Sato N, Mori Y, Minagawa N, Tamura T, Shibao K, Higure A and Yamaguchi K: Rapid postoperative reduction in prognostic nutrition index is associated with the development of pancreatic fistula following distal pancreatectomy. Pancreatology 14: 216-220, 2014.

This work is licensed under a Creative Commons Attribution-NonCommercial-NoDerivatives 4.0 International (CC BY-NC-ND 4.0) License. 